\title{
Traços de Personalidade Psicopática e a associação com Empatia e Contágio Emocional
}

\author{
Allana Gessiele Mello da Silva \\ Bruna Gomes Mônego \\ llana Andretta
}

\section{RESUMO}

As pesquisas empíricas sobre psicopatia têm enfatizado o modelo dimensional da personalidade na sua compreensão, possibilitando assim estudos na população em geral. A literatura atual tem identificado prejuízos na empatia dos indivíduos com traços de psicopatia, vista como um construto multidimensional com componentes afetivos e cognitivos, e no contágio emocional, um processo mais primitivo e filogenético. Entretanto, pesquisas sobre o tema na população em geral ainda são escassas. 0 objetivo deste estudo foi avaliar a associação de traços de psicopatia, empatia e contágio emocional em população geral. A amostra foi composta por 284 participantes, com idade média de 24,6 anos (DP=8,37). Instrumentos utilizados foram o Instrumento de Autorrelato para avaliar traços de psicopatia, o Interpersonal Reactivity Index para avaliar empatia e o Emotional Contagion Scale para avaliar contágio emocional. Foram encontradas associações negativas entre empatia e traços de psicopatia, associações negativas entre contágio emocional e empatia e associações positivas entre contágio emocional e traços de psicopatia. Os resultados indicam que os traços de psicopatia estão associados com menores níveis de empatia e contágio emocional, indicando que existem características de dessensibilização emocional, e que a empatia e o contágio emocional estão fortemente associados, sugerindo serem processos complementares.

Palavras-chave: empatia; contágio emocional; cognição social; psicopatia; traços de psicopatia.

\section{ABSTRACT}

\section{Psychopathic personality traits and the association with empathy and emotional contagion}

Empirical research on psychopathy has emphasized the dimensional model of personality in its understanding, thus enabling studies in the general population. Current literature has identified empathy impairments in individuals with psychopathic traits, seen as a multidimensional construct with affective and cognitive components, and in emotional contagion, a more primitive and phylogenetic process. Nevertheless, research on this subject in the general population is still scarce. This study aims to evaluate the association of psychopathic traits, empathy and emotional contagion in general population. The sample was composed of 284 participants (mean age 24.6 years; SD $=8.37$ ). The instruments used were a self-report instrument to evaluate psychopathic traits, the Interpersonal Reactivity Index to evaluate empathy and the Emotional Contagion Scale to assess emotional contagion. Negative associations were found between empathy and psychopathic traits and between emotional contagion and empathy, such as positive associations between emotional contagion and psychopathic traits. Results show that psychopathic tendencies are associated with lower levels of empathy and emotional contagion, indicating the existence of emotional desensitization characteristics, and that empathy and emotional contagion are strongly associated, suggesting that both are complementary processes.

Keywords: empathy; emotional contagion; social cognition; psychopathy; psychopathy traits.

\section{Sobre os Autores}

A. G. M. S.

orcid.org/ 0000-0002-3086-

2109

Universidade do Vale do Rio dos Sinos (UNISINOS) - São

Leopoldo, RS

allanagm@hotmail.com

B. G. M.

orcid.org/0000-0001-7655-7188

Centro Universitário Cenecista de Osório (UNICNEC) - Osório, RS

brunamonego@hotmail.com

I. A.

orcid.org/0000-0002-5537-5120 Universidade do Vale do Rio dos Sinos (UNISINOS) - São

Leopoldo, RS

ilana.andretta@gmail.com

\section{Direitos Autorais}

Este é um artigo de acesso aberto e pode ser reproduzido livremente, distribuído, transmitido ou modificado, por qualquer pessoa desde que usado sem fins comerciais. 0 trabalho é disponibilizado sob a licença Creative Commons CCBY-NC.

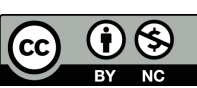




\section{W'INTERACÃO EM ET PSICOLOGIA}

A literatura aponta que o estudo da psicopatia se iniciou no século XIX por meio do médico francês Phillipe Pinel, o primeiro a publicar uma descrição do que hoje se assemelha à compreensão desse quadro, e apresentar ao mundo científico um transtorno que, apesar de os indivíduos não exibirem sintomas psicóticos, tinham uma conduta imoral e extravagante (Vaughn \& Howard, 2005). Posteriormente, alguns autores se dedicaram a estudar o tema, entretanto, foi Harvey Cleckley que identificou um conjunto de 16 características da personalidade psicopática: 1) charme superficial e inteligência; 2) ausência de delírios; 3) ausência de manifestações psiconeuróticas; 4) não-confiabilidade; 5) tendência à mentira; 6) falta de remorso ou vergonha; 7) comportamento antissocial; 8) juízo empobrecido; 9) egocentrismo patológico; 10) pobreza de reações afetivas; 11) perda de insight; 12) déficit de reciprocidade nas relações interpessoais; 13) comportamento fantasioso e nãoconvidativo; 14) ameaças de suicídio; 15) vida sexual impessoal e trivial; 16) falha em seguir um plano de vida (Barlow \& Durand, 2008; Hauck Filho, Teixeira \& Dias, 2009). Foram os estudos de Cleckeley que embasaram o estudo pioneiro de Robert Hare, o principal pesquisador em psicopatia da atualidade (Barlow \& Durand, 2008; Vaughn \& Howard, 2005).

Na perspectiva de Hare e Neumann (2008), a psicopatia apresenta quatro dimensões subjacentes: interpessoal, afetiva, estilo de vida e antissocial. A dimensão interpessoal está relacionada com superficialidade, manipulação, autoestima exagerada e mentira patológica. A afetiva indica ausência de remorso, afeto superficial, falta de empatia e falta de autorresponsabilização pelos próprios atos. 0 estilo de vida está relacionado à necessidade de estímulo, impulsividade, irresponsabilidade, parasitismo em relação aos outros e falta de objetivos realistas. Por fim, a dimensão antissocial refere-se à dificuldade no controle do comportamento, problemas comportamentais precoces, delinquência na juventude, versatilidade criminosa e revogação de liberdade condicional (Hauck Filho et al., 2009; Hare \& Neumann, 2008).

Compreende-se que a psicopatia é um termo complexo e amplo. Na tentativa de aprimorar e facilitar a concordância e precisão dos diagnósticos entre os profissionais desenvolveu-se, a partir dos estudos sobre a psicopatia, o diagnóstico do Transtorno da Personalidade Antissocial (TPA), presente no Manual Diagnóstico e Estatístico de Transtornos Mentais 5 (DSM 5), que enfoca apenas aspectos comportamentais antissociais (APA, 2014; Arrigo \& Shipley, 2001; Hauck Filho et al., 2009). O TPA caracteriza-se por um padrão difuso de desconsideração e violação dos direitos das outras pessoas, tendo os seguintes critérios diagnósticos: 1) fracasso em se ajustar às normas sociais e legais; 2) tendência à falsidade, indicada por mentiras repetidas e trapaças; 3) impulsividade ou fracasso em fazer planos para o futuro; 4) irritabilidade e agressividade, apresentando repetidas agressões físicas; 5 ) descaso pela segurança de si ou de outros; 6) irresponsabilidade reiterada, indicada por falha em manter uma conduta consistente no trabalho ou honrar obrigações financeiras; 7) ausência de remorso, caracterizada pela indiferença ou racionalização em relação a fazer mal para outras pessoas (APA, 2014). Entretanto, esse diagnóstico não permite a distinção entre o indivíduo que apresenta apenas comportamento antissocial daquele que também apresenta as características das esferas afetiva, interpessoal e de estilo de vida da psicopatia. Somente a presença dos comportamentos antissociais não é suficiente para o diagnóstico dessa (Hauck Filho et al., 2009; Kosson, Lorenz \& Newman, 2006). Dessa forma, a psicopatia tem sido estudada por apresentar-se como uma descrição mais completa e complexa do que o TPA.

Considerando a psicopatia como um transtorno da personalidade, cabe definir que personalidade pode ser compreendida como um conjunto integrado de características psíquicas, fatores físicos, biológicos e socioculturais, unindo aspectos inatos e adquiridos que constituem o indivíduo (Barlow \& Durand, 2008), permeando a relação dele com 0 ambiente. Porém, características inflexíveis e mal adaptativas da personalidade podem constituir sintomas de transtornos de personalidade (APA, 2014).

Pesquisas empíricas sobre a personalidade têm enfatizado o modelo dimensional na compreensão da psicopatia, operacionalizado pelo modelo triárquico da psicopatia, que a avalia em três grandes dimensões, o saber, a ousadia (boldness), a malvadez (meanness) e a desinibição (dishinibition) (Patrick, Fowles \& Krueger, 2009). Tal modelo compreende a personalidade como parte de um continuum de características, de acordo com o grau de presença delas. Embora alguns autores ainda trabalhem com a concepção categorial de presença ou ausência da psicopatia, a concepção dimensional vem ganhando força (Vasconcellos \& Vasconcellos, 2012). Existem evidências empíricas favoráveis à utilização da avaliação dimensional desse quadro, sugerindo que a perspectiva categórica pode não ser a melhor forma de avaliação (Edens, Marcus \& Vaughn, 2011; Walters et al., 2007; Walters, Brinkley, Magaletta \& Diamond, 2008). Além disso, por trabalhar com uma perspectiva de traços, a compreensão dimensional da psicopatia permitiu que fossem realizadas pesquisas na população em geral, investigando o grau desses traços ao invés de avaliar apenas amostras de indivíduos institucionalizados (Hauck Filho et al., 2009). 


\section{W'INTERACÃO EM ET PSICOLOGIA}

Considerando que um dos aspectos mais proeminentes da descrição da psicopatia é a desconsideração do outro, a literatura tem identificado prejuízos relacionados à cognição social desses indivíduos. Tal conceito compreende um conjunto de processos cognitivos usados para decodificar e codificar o mundo social, incluindo processamentos sobre pessoas, o próprio self, e sobre as normas e procedimentos, podendo esse processamento ser automático ou em níveis com algum controle (Monteiro \& Neto, 2011). Dentro desse construto, a empatia tem papel importante quando se trata da compreensão da vivência social dos indivíduos, pois é essencial na interação entre eles.

Inicialmente a empatia foi descrita por Titchener, em 1909, como a capacidade de conhecer a consciência de outra pessoa, e por meio da imitação interna, pensar de maneira análoga a ela (Sampaio, Camino \& Roazzi, 2009). Posteriormente, o tema foi introduzido na clínica psicológica como uma característica fundamental para a terapia, compreendendo-a como algo além de um entendimento "exterior" sobre os pensamentos e sentimentos da outra pessoa, implicando sensibilização, apreensão e compreensão dos estados internos desta e sem fazer nenhum julgamento de valor (Sampaio et al., 2009). Atualmente, a empatia ainda não tem uma definição única, mas sim um campo conceitual em construção. Numa tentativa de conceitualização é possível definir a empatia como uma capacidade para compreender os sentimentos e emoções do outro, reconhecendo-o como ser semelhante (López, Filippetti \& Richaud, 2014; Sampaio et al., 2009). Modelos integradores da empatia concebem-na como um construto multidimensional, que inclui processos afetivos, que são um sistema básico, e cognitivos, que são mais avançados e requerem tomada de perspectiva. Contudo, esses processos não são dissociados, mesmo que diferentes áreas e estruturas cerebrais sejam responsáveis por cada um (Filippetti, López \& Richaud, 2012; Shamay-Tsoory, AharonPeretz \& Perry, 2009).

0 componente cognitivo da empatia refere-se à capacidade do ser humano de entender a experiência emocional do outro a partir da tomada de perspectiva. Desta forma, na experiência empática inferem-se os sentimentos, pensamentos e emoções da pessoa observada, mas sem necessariamente sentir a mesma emoção que ela (Cuff, Brown, Taylor \& Howat, 2014; Falcone et al., 2008). A capacidade de tomada de perspectiva se dá por meio da interrelação entre a autoconsciência e a consciência do outro que é diferente do eu, e por meio da experiência do self, que permite o desenvolvimento do conhecimento inferencial do estado mental do outro (Decety \& Jackson, 2004; Falcone et al., 2008).
Em relação à empatia afetiva, a experiência emocional do self é eliciada pelos estímulos emocionais que o outro provoca (Cuff et al., 2014). É importante salientar que nessa experiência não há confusão entre o self e o outro, ou a experiência da mesma emoção do outro, mas sim a vivência da própria emoção a partir do entendimento da emoção do outro (Falcone et al., 2008).

Quando há uma identificação inata do self com o sentimento do outro sem um processo consciente, trata-se de um contágio emocional, sendo esse um fenômeno mais primitivo e filogenético do ser humano, necessário para o desenvolvimento da empatia. Esse processo se dá por meio das expressões faciais, vocalizações, posturas e movimentos, convertendo tudo isto em uma experiência emocional simultânea e do próprio self, semelhante àquela experienciada pelo outro (Gouveia et al., 2007; Hatfield et al., 1993; López et al., 2014). É importante frisar que contágio emocional não é o mesmo que empatia, sendo o primeiro um fenômeno caracterizado por uma forma simples de experienciação e compartilhamento de afeto, não dependendo de processos cognitivos de ordem superior, ainda que o mesmo seja base para fenômenos cognitivos mais complexos, como a empatia. Desta forma, o contágio emocional é o nível primário da resposta empática (López et al., 2014; Sturm et al., 2013).

Considerando o exposto, seria possível que indivíduos com altos níveis de traços de psicopatia apresentassem prejuízos na empatia e no contágio emocional? Alguns estudos demonstraram correlações significativas entre traços de psicopatia e empatia (Brook \& Kosson, 2013; Khvatskaya \& Lenzenweger, 2016; Lishner et al., 2012; Lishner, Hong, Jiang, Vitacco \& Neumann, 2015; Luckhurst, Hatfield \& Gelvin-Smith, 2017; Mahmut, Homewood \& Stevenson, 2008; SearaCardoso, Neumann, Roiser, Mccrory \& Viding, 2012) e contágio emocional (Lishner et al., 2012; Lishner et al., 2015; Luckhurst et al., 2017). Entretanto, esses resultados variam quanto à magnitude e direção da correlação, amostras e instrumentos adotados, e a inclusão de uma medida de contágio emocional é menos frequente.

Os resultados indicam, por exemplo, que os participantes com altos níveis de psicopatia apresentam respostas empáticas mais fracas quando são apresentadas situações de experiência emocional de medo ou de tristeza (Khvatskaya \& Lenzenweger, 2016; Mahmut, Homewood \& Stevenson, 2008; Seara-Cardoso, Neumann, Roiser, Mccrory \& Viding, 2012), além de pior precisão empática para as mesmas emoções (Brook \& Kosson, 2013). Encontrou-se evidência de que maiores níveis de psicopatia estariam relacionados a maior empatia afetiva; especificamente, a característica de insensibilidade emocional estaria associada 


\section{N"INTERACÃO EM ET PSICOLOGIA}

a uma menor empatia afetiva e menor contágio emocional para as emoções tristeza, raiva e medo (Lishner et al., 2015). Essas pesquisas indicam as discordâncias entre os resultados, assim como a necessidade do desenvolvimento de estudos com os construtos que compõem a empatia e contágio emocional associados à psicopatia.

Embora seja crescente o número de estudos internacionais sobre traços psicopáticos na população não carcerária e as relações com processos empáticos, essa realidade ainda não ocorre no Brasil, sendo esta evidenciada pelos poucos estudos na literatura desse país com tais populações. Desta forma, evidencia-se a importância de estudos que possam mostrar a compreensão do processo empático, incluindo o contágio emocional, em indivíduos da população em geral que apresentam traços de psicopatia, a fim de encontrar possíveis associações. Portanto, o objetivo desse estudo foi avaliar a associação de traços de psicopatia com empatia e contágio emocional na população geral, não institucionalizada.

\section{MÉTODO}

\section{PARTICIPANTES}

A amostra foi selecionada por conveniência adotando-se critérios de exclusão por idade (não podendo participar menores de 18 anos) e escolaridade (mínimo, ensino médio completo). Participaram deste estudo 284 indivíduos, sendo $204(71,8 \%)$ do sexo feminino e 80 (28,2\%) do sexo masculino, selecionados por conveniência. A idade dos participantes variou entre 18 e 65 anos $(M=24,6$ anos; $D P=$ 8,37 anos). Sobre a escolaridade, o ensino superior incompleto $(n=273 ; \%=96,1)$ foi predominante. A situação conjugal dos participantes foi declarada como solteiros $(n=118 ; \%=41,7)$, namorando $(n=101 ; \%=35,7)$ e casados $(n=63 ; \%=22,3)$. A classe econômica teve variação, ficando os participantes distribuídos da seguinte forma: classe $A \mathrm{n}=92$ $(32,4 \%)$, classe $B n=149(52,4 \%)$, classe $C n=42(14,8 \%)$.

\section{INSTRUMENTO}

\section{Questionário de dados sociodemográficos}

Foi desenvolvido pelas autoras do presente estudo no modelo de entrevista estruturada para identificar informações sobre idade, escolaridade, religião, situação conjugal e classe econômica, por meio de perguntas objetivas.

\section{Instrumento de Autorrelato para Avaliar Traços de Psicopatia}

O Instrumento de Autorrelato para Avaliar Traços de Psicopatia (Hauck Filho, Salvador-Silva \& Teixeira, 2015) foi desenvolvido em contexto brasileiro para medir traços de psicopatia na população geral, utilizando Métodos de Teoria de Resposta ao Item e análise fatorial ordinal. 0 instrumento é composto por 60 itens que avaliam três dimensões da psicopatia: Egocentrismo Maquiavélico, que abarca aspectos de insensibilidade, manipulação e egocentrismo; Descontrole, que engloba aspectos de impulsividade e comportamentos antissociais; e Audácia, que inclui aspectos de dominância social, resiliência e ausência de medo. É respondido por meio de escala do tipo Likert com quatro pontos, variando entre "definitivamente não" e "definitivamente sim". Os resultados do estudo de desenvolvimento da escala apresentaram propriedades psicométricas satisfatórias para o modelo trifatorial. Os valores da consistência interna das dimensões Egocentrismo Maquiavélico, Descontrole e Audácia foram de $0,84,0,84$ e 0,69, respectivamente. No presente estudo, obteve-se os valores $0,75,0,81$ e 0,66 , respectivamente. 0 Global Fit Index, um importante índice de ajuste, foi de 0,96 e a variância explicada foi de $33,71 \%$. Ainda, foram reportados valores adequados de fidedignidade dos itens $(0,99$ a 1,00) e das pessoas $(0,64$ a 0,80$)$ para as três dimensões e os itens apresentaram boa capacidade discriminativa. Evidências de validade convergente também foram relatadas (Hauck Filho et al., 2015).

\section{Interpersonal Reactivity Index [IRI]}

O IRI (Davis, 1983; Sampaio, Guimarães, Camino, Formiga \& Menezes, 2011) foi desenvolvido para avaliar a empatia. É um instrumento de autorrelato composto por 26 itens e quatro dimensões, a saber: (1) Consideração Empática, que avalia a motivação para ajudar outras pessoas, ou seja, motivação pró-social; (2) Tomada de Perspectiva, que se refere à habilidade de se colocar no lugar de outras pessoas, imaginando o que elas pensam ou sentem; (3) Angústia Pessoal, que se relaciona ao incômodo ou ansiedade produzidas no self ao se deparar com situações tensas ou de emergência; e (4) Fantasia, que inclui a tendência de transpor a si mesmo imaginativamente, colocando-se no lugar de personagens fictícios. Alguns autores classificam as dimensões Tomada de Perspectiva e Fantasia como empatia cognitiva e Angústia Pessoal e Consideração Empática como empatia emocional. O IRI é respondido por meio de uma escala do tipo Likert de cinco pontos que tem como pontos extremos "Não me descreve bem" e "Descreve-me muito bem". Em relação ao estudo de adaptação da escala para o Brasil, os resultados indicaram propriedades psicométricas satisfatórias para o modelo tetrafatorial. Os índices obtidos na análise fatorial confirmatória com o método de estimação 


\section{H NTERAC̄öEM ET PSICOLOGIA}

Maximum Likelihood foram adequados, a saber: a razão entre qui-quadrado e os graus de liberdade $\left(x^{2} / g . l\right.$. = 2,84), o Adjusted Goodness-of-Fit Index (AGFI = 0,91), o Goodness-ofFit Index (GFI = 0,94), a Raiz Quadrada Média Residual (RMR = 0,05), o Expected Cross-Validation Index (ECVI = 1,97), o Consistent Akaike Information Criterion (CAIC = 999,56), o Root-Mean-Square Error of Approximation (RMSEA $=0,01$ ) e o Comparative Fit Index $(\mathrm{CFI}=0,99)$. Ainda, todas as saturações se mantiveram dentro do intervalo esperado de 0 a 1 , excluindo problemas de estimação, e foram estatisticamente diferentes de zero $(t>1,96, p<0,05)$. A análise de consistência interna das dimensões Fantasia, Consideração Empática, Angústia Pessoal, Tomada de Perspectiva e da escala total resultou nos valores $0,82,0,72,0,766,0,719$ e 0,85 , respectivamente, enquanto que no presente estudo foram observados valores de $0,79,0,85,0,79,0,80$ e 0,86 . Não foram reportados resultados de fidedignidade (Sampaio et al., 2011).

\section{Emotional Contagion Scale [ECS]}

A ECS (Doherty, 1997; Gouveia et al., 2007) foi desenvolvida por Doherty (1997) para avaliar a suscetibilidade ao contágio emocional e sua versão em português foi chamada de Escala de Contágio Emocional. 0 instrumento de autorrelato é composto por 18 itens $(a=0,84)$ que avaliam o contágio emocional de cinco emoções: Amor $(a=0,60)$, Felicidade $(a=0,10)$, Medo $(a=0,40)$, Raiva $(a=$ $0,55)$ e Tristeza $(\alpha=0,65)$, além de conter as dimensões Emoções Positivas $(a=0,73)$ e Emoções Negativas $(a=0,76)$. Os valores de consistência interna reportados foram obtidos no presente estudo. 0 instrumento é respondido por meio de escala do tipo Likert com quatro pontos, variando os pontos extremos entre "Sempre" e "Nunca". O estudo de adaptação da escala para o Brasil (Gouveia et al., 2007) realizou análise fatorial confirmatória empregando o método de estimação Maximum Likelihood. Os pesquisadores testaram alguns modelos e optaram pela estrutura com cinco fatores de primeira ordem, que seriam as emoções básicas, e dois fatores de segunda ordem, Emoções Positivas, formada pelos itens de Amor e Felicidade; e Emoções Negativas, formada pelos itens de Tristeza, Raiva e Medo. Tal modelo apresentou índices satisfatórios (Qui-quadrado $\left[x^{2}\right](84)=238,69, \mathrm{p}<$ $0,001, x^{2} /$ g.I. $\left.=2,84, \mathrm{GFI}=0,90, \mathrm{AGFI}=0,86, \mathrm{RMSEA}=0,079\right) \mathrm{e}$ as saturações foram estatisticamente diferentes de zero $(t>$ $1,96, p<0,05)$. Outras informações sobre a análise fatorial confirmatória não foram reportadas no artigo, mas os autores informaram disponibilizá-las mediante solicitação. Os índices de consistência interna encontrados foram: Amor $(a=0,56)$, Felicidade $(a=0,52)$, Medo $(a=0,52)$, Raiva $(a=0,61)$, Tristeza $(a=0,60)$, Emoções Positivas $(a=0,64)$ e Emoções Negativas $(a=0,73)$ (Gouveia et al., 2007). Assim como em outros estudos brasileiros (Gondim, Gouveia, Alberton, et al., 2014; Gondim, Gouveia, Simões, et al., 2014), optou-se pelo uso de pontuações diferentes para os fatores de primeira e de segunda ordem. É importante salientar que, diferentemente das demais escalas do estudo, a ECS tem o somatório de suas dimensões invertido, sendo a maior pontuação indicadora de menor contágio emocional.

\section{PROCEDIMENTOS}

O presente estudo foi aprovado pelo Comitê de Ética em pesquisa da Universidade do Vale do Rio dos Sinos, sob o número $15 / 255$. Os indivíduos foram convidados a participar, os que aceitaram e concordaram com a pesquisa receberam e assinaram o Termo de Consentimento Livre e Esclarecido (TCLE).

A coleta de dados foi feita presencialmente nas salas de aula da Universidade do Vale do Rio dos Sinos, com autorização prévia dessa instituição. Com o consentimento dos professores, todos(as) os(as) alunos(as) foram convidados a participar voluntariamente do estudo. 0 convite aconteceu em salas de aulas de cursos diversos, na área de saúde, humanas e exatas, a fim de que a amostra tivesse variabilidade. Também foi pedido aos participantes indicações de outros indivíduos, fora da universidade, que poderiam responder ao questionário, se configurando uma amostra por conveniência.

O presente estudo é descritivo e associativo de caráter quantitativo e transversal (Gil, 2010). Os dados foram analisados por meio do programa estatístico SPSS (versão $20)$, considerando o nível de significância de $5 \%(p \leq 0,05)$. Inicialmente foram feitas análises descritivas de tendência central e de dispersão, e a distribuição das variáveis intervalares foi investigada por meio do teste KolmogorovSmirnov. Posteriormente, calculou-se a Correlação de Pearson, avaliando possíveis associações entre as três dimensões da escala de traços de psicopatia, com os cinco fatores de primeira ordem e os dois fatores de segunda ordem da escala ECS, e também com as quatro dimensões do IRI. A magnitude da correlação é indicada pelo valor de $r$ (coeficiente de correlação) e pode ser interpretada como fraca quando os valores estão entre 0,1 e 0,3; moderada entre 0,4 e 0,6 ; forte entre 0,7 e 0,9 ; e perfeita quando atinge 1 (Dancey \& Reidy, 2013).

\section{RESULTADOS}

De modo geral, as análises demonstraram que os traços de psicopatia foram associados negativamente com a 


\section{- H. INTERACÃO EM LF PSICOLOGIA}

empatia e positivamente com o contágio emocional. A maioria dos resultados estatisticamente significativos apresentaram uma magnitude que pode ser considerada fraca. As que podem ser interpretadas como moderadas foram observadas na relação do traço Audácia com as dimensões Angústia Pessoal, Medo e Emoções Negativas. Os valores das correlações de Pearson estão descritos na Tabela 1.

Tabela 1. Correlações entre Traços de Psicopatia, IRI e ECS

\begin{tabular}{|c|c|c|c|}
\hline & \multicolumn{3}{|c|}{ Traços de Psicopatia } \\
\hline & Descontrole & Audácia & Egocentrismo \\
\hline \multicolumn{4}{|l|}{$|\mathrm{R}|$} \\
\hline Fantasia & $-0,258 * \star \star$ & $-0,245^{\star \star *}$ & 0,037 \\
\hline Angústia Pessoal & 0,102 & $-0,458 * \star \star$ & 0,020 \\
\hline Consideração Empática & $-0,073$ & $-0,238 * \star \star$ & $-0,305^{\star \star \star}$ \\
\hline Tomada de Perspectiva & $-0,193$ ** & $-0,150$ & 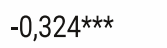 \\
\hline \multicolumn{4}{|l|}{ ECS } \\
\hline Amor & $0,153^{\star \star}$ & 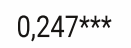 & $0,172^{\star \star}$ \\
\hline Felicidade & $0,138^{*}$ & $0,196^{\star \star}$ & $0,247^{\star \star \star}$ \\
\hline Medo & 0,043 & $0,411 \star \star \star$ & 0,084 \\
\hline Raiva & $-0,066$ & $0,345^{\star \star \star}$ & 0,085 \\
\hline Tristeza & 0,081 & $0,280^{\star \star \star}$ & $0,161^{* *}$ \\
\hline Emoções positivas & $0,169 * *$ & $0,260 \star \star \star$ & 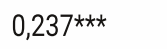 \\
\hline Emoções negativas & 0,026 & $0,419 * * *$ & $0,138 *$ \\
\hline
\end{tabular}

Nota. IRI= Interpersonal Reactivity Index; ECS= Emotional Contagion Scale; ${ }^{\star} p<0,05 ; \star \star p p>0,01 ; * \star x p>0,001$

\section{DISCUSSÃO}

Tendo em vista que o presente artigo se propôs a avaliar possíveis associações entre o processo empático, incluindo contágio emocional, com traços de psicopatia em indivíduos da população em geral, não institucionalizada, os resultados indicaram a presença de traços de psicopatia na amostra estudada e a existência de sua associação com empatia e contágio emocional. Dessa forma, foi possível demonstrar que os resultados desse estudo dão suporte à hipótese contínua (e não categórica) da compreensão da psicopatia.

Verificou-se que a dimensão Audácia, que inclui características de dominância social, ausência do medo e resiliência, esteve associada com níveis mais baixos de Consideração Empática, Fantasia e, principalmente, de Angústia Pessoal, mas não com Tomada de Perspectiva. A hipótese é que indivíduos com esse traço experienciem a empatia e avaliem o sofrimento do outro de forma mais cognitiva e racional. Desta forma, observa-se a redução no que se refere à capacidade de resposta ao estado emocional de terceiros, assim como pouca atenção em relação às necessidades destes. Essas características podem estar relacionadas à dificuldade na avaliação do estado emocional dos demais indivíduos (Brook \& Kosson, 2013; Falcone et al., 2008). Os resultados de estudo feito com 124 homens adultos da população em geral que avaliou a associação de psicopatia, empatia e moralidade indicaram que indivíduos com altos níveis de psicopatia apresentaram respostas empáticas mais fracas quando foram apresentados a situações de experiência emocional de medo ou tristeza (Seara-Cardoso et al., 2012). Outro estudo, com 101 estudantes universitários (27 homens, 74 mulheres), revelou que o grupo com maior nível de psicopatia apresentou menos empatia afetiva do que o grupo com menor nível de psicopatia. Também foi encontrada correlação negativa entre psicopatia e empatia geral (Mahmut et al., 2008).

Conforme esperado, a dimensão Egocentrismo Maquiavélico da psicopatia apresentou correlação negativa com os fatores Consideração Empática e Tomada de Perspectiva. Isso indica que, nesta amostra, os indivíduos com mais características de insensibilidade, manipulação e egocentrismo apresentaram menor capacidade de se colocar no lugar de outras pessoas, imaginando o que elas pensam ou sentem, menos comportamentos pró-sociais, maior preocupação consigo mesmo e com os ganhos que podem obter usando o outro e um maior distanciamento emocional. Isso pode ser explicado por meio da compreensão de que indivíduos com traços de Egocentrismo Maquiavélico não sentem necessidade de reparação ou cuidado por não perceberem suas atitudes como prejudiciais aos outros, indicando dificuldades interpessoais e afetivas (Vasconcellos \& Vasconcellos, 2012).

Em relação à associação entre traços de psicopatia e contágio emocional nesta amostra, os resultados demonstraram que quanto maior o nível de traços de psicopatia, menor é o contágio emocional, tanto pelas emoções negativas quanto pelas positivas. Apesar disso, os traços de psicopatia referentes às características de insensibilidade, manipulação e egocentrismo não apresentaram associação com contágio pelas emoções medo e raiva, indicando uma menor responsividade emocional a essas emoções especificamente. Resultado semelhante foi reportado por um estudo que apontou que 


\section{MI* INTERACÃO EM LF PSICOLOGIA}

características afetivas-interpessoais da psicopatia estão relacionadas a respostas empáticas mais fracas e frieza emocional, mais especificamente em relação à emoção medo, indicando menor propensão à sentir preocupação pelos outros em situações que despertam essas emoções. (Seara-Cardoso et al., 2012). Especificamente, a literatura mostra que indivíduos com psicopatia apresentam empatia afetiva; entretanto, o contágio emocional principalmente quando se refere às emoções negativas, evidenciando insensibilidade emocional (Lishner et al., 2015).

Uma maior dificuldade em sentir a emoção a partir da experiência do outro torna os indivíduos menos sensíveis e menos propensos a apresentar comportamentos empáticos (Lishner et al., 2015; Wai \& Tiliopoulos, 2012). Desta forma, acredita-se que níveis mais baixos de contágio emocional podem facilitar o desenvolvimento de traços acentuados de psicopatia e níveis mais baixos de empatia (López et al., 2014; Vasconcellos \& Gauer, 2004). As características psicopáticas de manipulação, frieza emocional e impulsividade, somadas à baixa ansiedade e ao medo reduzido, resultam em déficits atencionais e cognitivos que intensificam os prejuízos no processamento emocional já presentes na psicopatia (Corr, 2010).

Complementarmente, na literatura há indícios de que indivíduos com mais traços de psicopatia apresentam dificuldade, em diferentes níveis, no reconhecimento de expressões emocionais faciais, de vocalizações e de posturas, além de uma tendência recorrente de desvalorização da emoção do outro. Tais aspectos podem explicar ou influenciar a redução do contágio emocional observada nesses indivíduos (Khvatskaya \& Lenzenweger, 2016; Vasconcellos, Salvador-Silva, Dias, Davóglio \& Gauer, 2014).

Neste sentido, Wai e Tiliopoulos (2012) apresentaram resultados semelhantes na pesquisa realizada com 139 estudantes, que teve por objetivo avaliar possíveis associações entre psicopatia e empatia cognitiva, afetiva e reconhecimento facial de emoções. Os resultados mostraram correlações negativas e significativas entre empatia emocional e psicopatia, déficits afetivos em indivíduos que apresentam traços de psicopatia e menor sensibilidade afetiva em relação a expressões faciais de emoções negativas e positivas. Aspectos neurológicos também podem estar associados aos prejuízos em questão. Diferenças em ativações cerebrais ou aspectos estruturais são observadas quando compara-se grupos com diferentes níveis de psicopatia. Um estudo realizado com indivíduos psicopatas malsucedidos, que são assim nomeados por terem sido aprisionados e legalmente condenados por suas ações, indicou que eles apresentaram déficits estruturais na amígdala, responsável por desempenhar um papel na coordenação emocional de respostas, podendo também estar relacionada à redução do contágio emocional (Yang, Raine, Colletti, Toga \& Narr, 2010).

Por outro lado, o contágio emocional teve poucas e fracas correlações com traços de psicopatia com características de impulsividade e comportamentos antissociais na amostra estudada, ou seja, a dimensão Descontrole. A literatura indica que essas características estão mais fortemente relacionadas à maior propensão à expressão de agressividade e possíveis falhas no controle inibitório em contextos de ativação emocional, mas não especificamente com déficits emocionais e empáticos (Verona, Sprague \& Sadeh, 2012). Esses dados evidenciam a importância de estudar traços de psicopatia em sua descrição mais completa e complexa, e não somente a partir dos comportamentos antissociais, o que limita a compreensão sobre o fenômeno (Hauck Filho, Teixeira \& Dias, 2012; Kosson et al., 2006).

Por fim, este estudo oferece indícios de que, nesta amostra, os indivíduos que apresentaram mais traços de psicopatia também apresentaram menor contágio emocional e empatia, sendo mais significativo em relação à empatia afetiva. Compreende-se que há indicativos da interrelação entre esses diferentes construtos na compreensão do desenvolvimento afetivo empático e dos traços de psicopatia. Os achados estão de acordo com o que a literatura vem apresentando e podem ser de valia para pesquisas futuras.

Apesar disso, o presente estudo apresentou limitações relativas a não ter abrangido uma amostra maior e selecionada aleatoriamente, por ter mais participantes do sexo feminino do que masculino, com escolaridade sendo quase em sua totalidade ensino superior incompleto. Além disso, não apresentou análises estatísticas mais sofisticadas pelo número amostral não ser adequado para tanto. Estudos futuros poderiam investigar possíveis relações de predição de empatia, utilizando o contágio emocional e os traços de psicopatia como variáveis independentes. Ainda, a inclusão de uma avaliação do reconhecimento de faces emocionais poderia fornecer dados mais precisos em relação ao contágio emocional e sua relação com traços de psicopatia.

\section{DECLARAÇÃO DE FINANCIAMENTO}

A pesquisa relatada no manuscrito foi realizada por financiamento próprio.

\section{DECLARAÇÃO DA CONTRIBUIÇÃO DOS AUTORES}

A.G.M.S. administração do projeto; A.G.M.S, B.G.M. e I.A. 


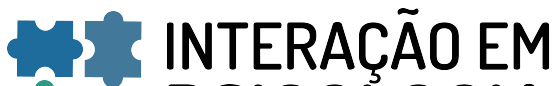 ET PSICOLOGIA}

análise formal dos dados; A.G.M.S, B.G.M. e I.A. conceitualização; A.G.M.S. investigação; A.G.M.S, B.G.M. e I.A. metodologia; A.G.M.S. redação - rascunho; A.G.M.S, B.G.M. e I.A. redação - revisão e edição; B.G.M. e I.A. supervisão; A.G.M.S. tabulação de dados; A.G.M.S, B.G.M. e I.A. validação; A.G.M.S, B.G.M. e I.A. visualização.

\section{DECLARAÇÃO DE CONFLITOS DE INTERESSE}

Os autores declaram que não há conflitos de interesse no artigo submetido.

\section{REFERÊNCIAS}

American Psychiatric Association (2014). Diagnostic and Statistical Manual of Mental Disorders (5a ed.). Washington, DC: American Psychiatric Association.

Arrigo, B., \& Shipley, S. (2001). The confusion over psychopathy (I): Historical considerations. International journal of Offender Therapy and Comparative Criminology, 3(45), 325-344. doi: 10.1177/0306624X01453005

Barlow, D. H., \& Durand, V. M. (2008). Transtornos da personalidade. In D. H. Barlow \& V. M. Durand, Psicopatologia: uma abordagem integrada (4a ed.) (pp. 502-541). São Paulo: Cengage Learning.

Brook, M., \& Kosson, D. S. (2013). Impaired cognitive empathy in criminal psychopathy: evidence from a laboratory measure of empathic accuracy. Journal of Abnormal Psychology, 122(1), 156-66. doi: 10.1037/a0030261

Corr, P. J. (2010). The psychoticism-psychopathy continuum: A neuropsychological model of core deficits. Personality and Individual Differences, 48(6), 695-703. doi: 10.1016/ j.paid.2009.12.023

Cuff, B. M. P., Brown, S. J., Taylor, L., \& Howat, D. J. (2014). Empathy: A review of the concept. Emotion Review, 8(2), 144-153. doi: $10.1177 / 1754073914558466$

Dancey, C. P., \& Reidy, J. (2013). Estatística sem matemática para psicologia (5a ed.) Porto Alegre: Penso Editora.

Davis, M. (1983). Measuring individual differences in empathy: Evidence for a multidimensional approach. Journal of Personality and Social Psychology, 44(1),113136.

Decety, J., \& Jackson, P. L. (2004). The functional architecture of human empathy. Behavioral and Cognitive Neuroscience Reviews, 3(2), 71-100. doi: 10.1177/1534582304267187

Doherty, R. W. (1997). The Emotional Contagion Scale: A measure of individual differences. Journal of Nonverbal Behavior, 21(2), 131-154. doi: 10.1023/A:1024956003661
Allana Gessiele Mello da Silva, Bruna Gomes Mônego e llana Andretta

Edens, J. F., Marcus, D. K., \& Vaughn, M. G. (2011). Exploring the taxometric status of Psychopathy among youthful offenders: is there a juvenile psychopath taxon? Law and Human Behavior, 35(1), 13-24. doi: 10.1007/s10979-0109230-8

Falcone, E. M. de O., Luz, R. C. M. da, Fernandes, C. S., D'augustin, J. F., Pinho, V. D. de, Ferreira, M. C., \& Sardinha, A. (2008). Inventário de empatia (i.e.): desenvolvimento e validação de uma medida brasileira. Avaliação Psicológica, 7(3), 321-334.

Filippetti, V. A., López, M. B., \& Richaud, M. C. (2012). Aproximación neuropsicológica al constructo de empatía: aspectos cognitivos y neuroanatómicos. Cuadernos de Neuropsicología Panamerican Journal of Neuropshychology, 6(1), 63-83.

Gil, A. C. (2010). Como elaborar projetos de pesquisa (5a ed.). São Paulo: Atlas.

Gondim, S. M. G., Gouveia, V. V., Alberton, G. D., Simões, A. C. A., de Morais, F. A., \& Díaz, M. I. H. (2014). Inteligência e Contágio Emocional: um estudo com trabalhadores brasileiros e angolanos. Interação em Psicologia, 18(2). doi: http://dx.doi.org/10.5380/psi.v18i2.30819

Gondim, S. M. G., Gouveia, V. V., Simões, A. C. A., Luna, A. F., Morais, F. A., Souza, R. V. L., \& Saveia, J. M. (2014). Constrangimento, contágio emocional e gestão: um estudo transcultural. Revista de Psicologia, 5(1).

Gouveia, V. V., Guerra, V. M., Santos, W. S. dos, Rivera, G. A., \& Singelis, T. M. (2007). Escala de Contágio Emocional: adaptação ao contexto brasileiro. PSICO, 38(1) 45-54.

Hare, R. D., \& Neumann, C. S. (2008). Psychopathy as a clinical and empirical construct. Annual Review of Clinical Psychology, 4(2), 217-246. doi: 10.1146/annurev.clinpsy. 3.022806 .091452

Hatfield, E., Cacioppo, J, L., \& Rapson, R. L. (1993). Emotional contagion. Current Directions in Psychological Sciences, 2(3), 96-100. doi: 10.1111/1467-8721.ep10770953

Hauck Filho, N. H., Salvador-Silva, R., \& Teixeira, M. A. P. (2015). Análise psicométrica preliminar de um instrumento de autorrelato para avaliar traços de psicopatia. Psico-USF, 20(2), 333-348. doi: 10.1590/141382712015200213

Hauck Filho, N. H., Teixeira, M. A. P., \& Dias, A. C. G. (2009). Psicopatia: o construto e sua avaliação. Avaliação Psicológica, 8(3), 337-346.

Hauck Filho, N. H., Teixeira, M. A. P., \& Dias, A. C. G. (2012). Psicopatia: uma perspectiva dimensional e não-criminosa do construto. Avances en Psicología Latinoamericana, 30(2), 317-327.

Khvatskaya, Y., \& Lenzenweger, M. F. (2016). Motor empathy in individuals with psychopathic traits: a preliminary study. Journal of Personality Disorders, 30(5), 613-632. 


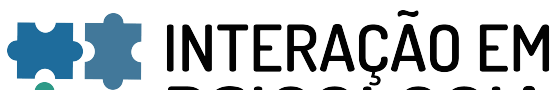 ET PSICOLOGIA}

Kosson, D. S., Lorenz, A. R., \& Newman, J. P. (2006). Effects of co-morbid psychopathy on criminal offending and emotion processing in male offenders with antisocial personality disorder. Journal of Abnormal Psychology, 115(4), 798-806. doi: 10.1037/0021-843X.115.4.798

Lishner, D. A., Hong, P. Y., Jiang, L., Vitacco, M. J., \& Neumann, C. S. (2015). Psychopathy, narcissism, and borderline personality: A critical test of the affective empathyimpairment hypothesis. Personality and Individual Differences, 86, 257-265. doi: 10.1016/j.paid.2015.05.036

Lishner, D. A., Vitacco, M. J., Hong, P. Y., Mosley, J., Miska, K., \& Stocks, E. L. (2012). Evaluating the relation between Psychopathy and affective empathy: two preliminary studies. International. Journal of Offender Therapy and Comparative Criminology, 56(8), 1161-1181. doi: 10.1177/0306624X11421891

López, M. B., Filippetti, V. A., \& Richaud, M. C. (2014). Empatía: desde la percepción automática hasta los procesos controlados. Avances en Psicología Latinoamericana, 32(1), 37-51. doi: 10.12804/apl32.1.2014.03

Luckhurst, C., Hatfield, E., \& Gelvin-Smith, C. (2017). Capacity for empathy and emotional contagion in those with psychopathic personalities. Interpersona, 11(1), 70-91. doi: 10.5964/ijpr.v11i1.247

Mahmut, M. K., Homewood, J., \& Stevenson, R. J. (2008). The characteristics of noncriminals with high psychopathy traits: Are they similar to criminal psychopaths? Journal of Research in Personality, 42(3), 679-692. doi: 10.1016/j.jrp. 2007.09.002

Monteiro, L. de C., \& Neto, M. R. L. (2011). Cognição social. In: L. F. Malloy-Diniz, D. Fuentes, P. Mattos \& N. Abreu (Orgs.), Avaliação Neuropsicológica (pp. 162-168). Porto Alegre: Artmed.

Patrick, C. J., Fowles, D. C., \& Krueger, R. F. (2009). Triarchic conceptualization of psychopathy: Developmental origins of disinhibition, boldness, and meanness. Development and Psychopathology, 21(3), 913-938. doi: 10.1017/ S0954579409000492

Sampaio, L. R., Camino, C. P. dos S., \& Roazzi, A. (2009). Revisão de aspectos conceituais, teóricos e metodológicos da empatia. Psicologia Ciência e Profissão, 29(2), 212-227.

Sampaio, L. R., Guimarães, P. R. B., Camino, C. P. dos S., Formiga, N. S., \& Menezes, I. G. (2011). Estudos sobre a dimensionalidade da empatia: tradução e adaptação do Interpersonal Reactivity Index (IRI). Psico, 42(1), 67-76.

Seara-Cardoso, A., Neumann, C., Roiser, J., McCrory, E., \& Viding, E. (2012). Investigating associations between empathy, morality and psychopathic personality traits in the general population. Personality and Individual Differences, 52(1), 67-71. doi: 10.1016/j.paid 2011.08.029
Shamay-Tsoory, S. G., Aharon-Peretz, J., \& Perry, D. (2009). Two systems for empathy: a double dissociation between emotional and cognitive empathy in inferior frontal gyrus versus ventromedial prefrontal lesions. Brain a Journal of Neurology, 132(3), 617-627. doi: 10.1093/brain/awn279

Sturm, V. E., Yokoyama, J. S., Seeley, W. W., Kramer, J. H., Miller, B. L., \& Rankin, K. P. (2013). Heightened emotional contagion in mild cognitive impairment and Alzheimer's disease is associated with temporal lobe degeneration. PNAS, 110(24), 9944-9949. doi: 10.1073/pnas. 1301119110

Vasconcellos, S. J. L., \& Gauer, G. J. C. (2004). A abordagem evolucionista do transtorno de personalidade anti-social. Revista de Psiquiatria do Rio Grande do Sul, 26(1), 78-85. doi: 10.1590/S0101-81082004000100011

Vasconcellos, S. J. L., Salvador-Silva, R., Dias, A. C., Davóglio, T. R., \& Gauer, G. (2014). Psicopatia e reconhecimento de expressões faciais de emoções: uma revisão sistemática. Psicologia: Teoria e Pesquisa, 30(2), 125-134. doi: 10.1590/ S0102-37722014000200001

Vasconcellos, C. T. de D. V., \& Vasconcellos, S. J. L. (2012). Quem eram os psicopatas? A história de alguns conceitos e suas implicações. In G. J. C. Gauer, S. J. L. Vasconcellos \& T. R. Davoglio (Orgs.). Adolescentes em conflito: violência, funcionamento antissocial e traços de psicopatia (pp. 13-34). São Paulo: Casa do Psicólogo, 2012.

Vaughn, M. G., \& Howard, M. O. (2005). The construct of psychopathy and its potential contribution to the study of serious, violent, and chronic youth offending. Youth Violence and Juvenile Justice, 3(3), 235-252. doi: $10.1177 / 1541204005276320$

Verona, E., Sprague, J., \& Sadeh, N. (2012). Inhibitory control and negative emotional processing in Psychopathy and Antisocial Personality Disorder. Journal of Abnormal Psychology, 121(2), 498-510. doi: 10.1037/a0025308

Wai, M., \& Tiliopoulos, N. (2012). The affective and cognitive empathic nature of the dark triad of personality. Personality and Individual Differences, 52(7), 794-799. doi: 10.1016/j.paid.2012.01.008

Walters, G. D., Brinkley, C. A., Magaletta, P. R., \& Diamond, P. M. (2008). Taxometric analysis of the Levenson SelfReport Psychopathy Scale. Journal of Personality Assessment, 90(5), 491-498. doi: $10.1080 / 00223890802248828$

Walters, G. D., Gray, N. S., Jackson, R. L., Sewell, K. W., Rogers, R., Taylor, J., \& Snowden, R. J. (2007). A Taxometric analysis of the Psychopathy Checklist: Screening Version (PCL:SV): Further evidence of dimensionality. Psychological Assessment, 19(3), 330 -339. doi: 10.1037/1040-3590.19.3.330 


\section{N"INTERACÃO EM \\ ET PSICOLOGIA}

Allana Gessiele Mello da Silva, Bruna Gomes Mônego e llana Andretta

Yang, Y., Raine, A., Colletti, P., Toga, A. W., \& Narr, K. L. (2010). Morphological alterations in the prefrontal cortex and the amygdala in unsuccessful psychopaths. Journal of Abnormal Psychology, 119(3), 546-554. doi: 10.1037/

Data de submissão: 23/01/2018 Primeira decisão editorial: 11/05/2018 Aceite em 13/09/2018 